\title{
Preliminary study of letrozole use for improving spermatogenesis in non-obstructive azoospermia patients with normal serum FSH
}

\author{
Giorgio Cavallini ${ }^{1}$, Giovanni Beretta ${ }^{2}$ and Giulio Biagiotti ${ }^{3}$
}

We investigated whether letrozole ( $2.5 \mathrm{mg} \mathrm{day}^{-1}$ ) improves sperm count in non-obstructive azoospermia (NOA) patients. Four men were included in this study, and they had folliculo-stimulating hormone and other hormone levels within the normal range and no varicoceles or chromosomal aberrations. These four patients were administered letrozole for 3 months. Sperm count, testicular volume, gonadotropin, testosterone (T) and estradiol (E2) blood levels were assessed before, during and 1 week after the suspension of treatment. All patients showed spermatozoa in their ejaculate, increased gonadotropin and T levels and lower E2 levels ( $P<0.05$ in all cases), when letrozole was administered. This suggests that letrozole treatment might improve sperm count in an NOA sub-population; however, more studies, including the proper controls, are needed to confirm its efficacy.

Asian Journal of Andrology (2011) 13, 895-897; doi:10.1038/aja.2011.44; published online 27 June 2011

Keywords: letrozole; medical treatment; non-obstructive azoospermia; spermatogenesis

\section{INTRODUCTION}

Azoospermia indicates the absence of spermatozoa in the ejaculate after two assessments of centrifuged semen. Its prevalence is about $1 \%$ in the general population and $10 \%-15 \%$ in infertile couples. ${ }^{1}$ Azoospermia is classified as obstructive (OA) or non-obstructive (NOA). NOA is defined by the clinical evidence of strongly affected spermatogenesis, and $\mathrm{OA}$ results from a seminal-tract obstruction. Men with OA typically have normal-sized testes, possible epididymal fullness and normal serum follicle-stimulating hormone (FSH) levels. Men with NOA present frequently with small testes and an elevated FSH level; however, a minority of these men have normal testicles and FSH levels within the normal range. ${ }^{2}$ Only men with hypogonadotropic-hypogonadism NOA or varicocele-associated NOA may yield spermatozoa in their ejaculate after treatment; however, the majority of men with NOA and normal or elevated FSH levels are only able to father children after surgical retrieval of the spermatozoa from the testicles, which is performed by testicular sperm extraction (TESE) or with fine-needle aspiration (FNA). TESE and FNA yield spermatozoa in $30 \%-60 \%$ of cases depending on the technique used. ${ }^{1}$

Patry et $\mathrm{al}^{3}$ recently reported one case of hypospermatogenesis (having FSH levels within the normal range), which was converted to active spermatogenesis after letrozole (Femara; Sanofi-Novartis, Paris, France) use. However, the patient remained azoospermic due to an obstruction in the epididymis that was not adequately assessed when surgery was performed. Saylam et al. ${ }^{4}$ recently treated $17 \mathrm{NOA}$ patients who had low $(<10)$ testosterone $(\mathrm{T}) /$ estradiol (E2) ratios with letrozole. Four of these men showed spermatozoa in their ejaculate after 6 months of therapy.
Letrozole is a member of a novel class of nonsteroidal, hormonetargeting agents used for breast cancer therapy. It reversibly inhibits aromatase, which is an enzyme that converts androgen precursors in adipose tissue to estrogen. ${ }^{5}$ To offer an alternative to surgery, letrozole's efficacy in restoring spermatogenesis needs to be confirmed in a larger, independent and well-controlled study of patients with NOA and normal FSH levels. In the present study, we tested the effect of letrozole treatment in four NOA with normal FSH levels to determine a possible effect on sperm count.

\section{PATIENTS AND METHODS}

This study began on 2 January 2010 and is still ongoing. We are still reporting our results to date to stimulate more research in this field. An azoospermia diagnosis was determined with two centrifuged semen samples collected after a 3- to 4 -day abstinence period. ${ }^{6}$ The Andros-Italia Institutional Review Board authorized the study, and the patients gave written informed consent for the 'off-label' administration of letrozole.

Clinical history, physical examination, bilateral scrotal duplex scans, blood hormonal levels (FSH, luteinizing hormone (LH), E2, $\mathrm{T}$ and prolactin (PRL)) and a genetic assessment ( $\mathrm{Y}$ microdeletion detection, screening for cystic fibrosis and karyotype) were performed for each patient. ${ }^{2}$ Testicular volume $\left(\mathrm{cm}^{3}\right)$ was measured by ultrasonography using the three-diameter technique ((length $\times$ width $\times$ height $) \times 0.71) .^{7}$ A differential diagnosis between NOA and OA was determined using the bilateral scrotal duplex examination in all patients. A peak systolic velocity of the testicular arteries that was greater than $9 \mathrm{~cm} \mathrm{~s}^{-1}$ was considered sufficient for an NOA diagnosis. ${ }^{2}$

${ }^{1}$ Andros-Italia, Outpatient Clinic of Ferrara, Via Mascheraio 46, Ferrara 44121, Italy; ${ }^{2}$ Centro Italiano Fertilità e Sessualità, Via della Fortezza 6 , Firenze 50129 , Italy and ${ }^{3}$ Andros Italia, Sede di Perugia, Via Martiri dei Lager 58, Perugia 06128, Italy

Correspondence: Dr G Cavallini (giorgiocavallini@libero.it)

Received: 25 December 2010; Revised: 23 February 2011; Accepted: 11 April 2011; Published online: 27 June 2011 
The preliminary status of this study, which involves only four patients and lacks controls, required us to test patients who were very similar to a case described by Patry et al.; ${ }^{3}$ therefore, only men with FSH blood levels within the normal range $\left(2-10 \mathrm{IU}^{-1}\right)$, a normal karyotype and no Y microdeletion and/or varicoceles were included. Four patients $(29,35,41$ and 44 years old) were tested. Two patients had previously undergone FNA in another infertility centre, and no spermatozoa were retrieved. Cytological examination of the samples indicated hypospermatogenesis, and these patients refused surgery and asked for the medical treatment for NOA.

They received letrozole $\left(2.5 \mathrm{mg}\right.$ day $\left.^{-1}\right)$ for 3 months because 64 days are required for the complete maturation of spermatozoa from the stem-cell stage. ${ }^{8}$ Additonally, $2.5 \mathrm{mg} \mathrm{day}^{-1}$ is the recommended dose for human use, which has been used in previous studies. ${ }^{4} \mathrm{FSH}$, LH, T, E2 and PRL levels were measured monthly during 3 months. Sperm count and testicular volume calculations were performed before and 3 months after the beginning of treatment to reduce the need for many examinations, which improved their compliance with the study. An identical assessment was performed 1 week after drug suspension.

Differences of FSH, LH, T, E2 and PRL levels before, in the course and after letrozole administration have been assessed with KruskalWallis test. ${ }^{9}$ Calculations have been performed using MATLAB program (http://www.mathworks.it). $P<0.05$ was considered statistically significant.

\section{RESULTS}

The results are presented in Table 1. No patient who was treated with letrozole remained azoospermic. The sperm concentration before treatment was 0 in both centrifuged semen samples (see section on 'Patients and methods') in all patients. Three months after treatment, sperm concentrations ranged from 40000 to 90 $000 \mathrm{ml}^{-1}$. Hormone levels were within the reference values before treatment in all patients. FSH, LH and T levels progressively significantly increased each month and were higher than the reference values $(P<0.05)$ during the course of treatment; however, E2 and
PRL levels significantly fell $(P<0.01)$ within the first month of letrozole treatment. All hormones returned to their respective reference values 1 week after drug suspension. Testicular volume always remained below the reference value. The ejaculated spermatozoa were cryoconserved for intracytoplasmic sperm injection procedures. Side effects included the loss of libido (all cases), cutaneous rashes (two cases) and nervousness (one case).

\section{CONCLUSIONS}

The results indicate that spermatogenesis might improve with letrozole administration for 3 months in men with NOA and normal serum levels of FSH, E2 and T. This small group of four patients represents a subgroup of men suffering from NOA.

All patients suffered losses of libido despite the supra-physiological levels of T, FSH and LH; however, in a study on the sexual behaviour of men with aromatase congenital deficiency, a loss of libido occurred even with supra-physiological levels of testosterone. This suggests that oestrogens could have a role in supporting libido. ${ }^{10}$

Letrozole was used in this study because indirect comparisons between letrozole and anastrozole suggest stronger evidence for the benefits of the use of letrozole in breast cancer treatment in postmenopausal women who require oestrogen-deprivation therapy. This is probably due to its stronger inhibition of aromatase. ${ }^{11}$ Additionally, this drug was used in other studies of spermatogenesis and NOA (Patry et al. ${ }^{3}$ and Saylam et al. ${ }^{4}$ ). We anticipate that our study may provide information to either confirm or refute the results reported by Patry et al. ${ }^{3}$ Although the results look promising, additional data and double-blind, prospective-controlled trials are mandatory to ascertain the efficacy of letrozole for NOA.

If an effect of letrozole on spermatogenesis and sperm count in an NOA sub-population can be substantiated in further studies, this effect is most likely from an increase in gonadotropin and androgen levels in the blood and a parallel decrease in E2 levels. Similarly, aromatase inhibitors are believed to increase sperm count, androgen and gonadotropin levels and to lower blood E2 levels in oligoasthenoteratospermia patients. ${ }^{12,13}$

Table 1 Sperm count, testicular volume and blood levels of FSH, LH, T, E2 and PRL before, during and after letrozole administration (2.5 $\left.\mathrm{mg} \mathrm{day}^{-1}\right)$.

\begin{tabular}{|c|c|c|c|}
\hline Variables examined & Before treatment $^{f}$ & At the end of treatment (third month) ${ }^{f}$ & One week after treatment suspension ${ }^{f}$ \\
\hline FSH $\left(I U I^{-1}\right)^{a}$ & $4.8(3.2-5.8)$ & $44.0(38.4-49.0)$ & $4.2(3.9-4.4)$ \\
\hline $\mathrm{LH}\left(\mid \mathrm{U} \mathrm{I}^{-1}\right)^{\mathrm{b}}$ & $4.25(3.2-4.8)$ & $14.25(2.6-16.1)$ & $4.20(3.4-5.0)$ \\
\hline $\mathrm{T}\left(\mathrm{ng} \mathrm{dl}^{-1}\right)^{\mathrm{c}}$ & $331.0(259.2-411.9)$ & $1117.0(924.6-1296.2)$ & $393.0(305.3-532.9)$ \\
\hline $\operatorname{PRL}\left(m \cup I^{-1}\right)^{d}$ & $10.5(8-14)$ & 0 & $9.5(7-11)$ \\
\hline $\mathrm{E} 2\left(\mathrm{pg} \mathrm{ml^{-1 }}\right)^{\mathrm{e}}$ & $40.5(28-54)$ & $0(0-6)$ & $38.0(26-55)$ \\
\hline T/E2 ratio & $7.7(7.0-11.3)$ & $\infty(\infty-550)$ & $9.3(8.7-16.1)$ \\
\hline $\begin{array}{l}\text { Sperm concentration (sperm } \\
\text { number, } \mathrm{ml}^{-1} \text { ) }\end{array}$ & 0 & 65000 (40 000-90 000) & $700(500-1000)$ \\
\hline WHO class a+b motile sperm (\%) & 0 & $8.7(6.2-12.6)$ & - \\
\hline Typical forms (strict criteria) (\%) & 0 & $2.4(1.0-4.3)$ & - \\
\hline Sperm volume (ml) & $2.6(2-4)$ & $2.9(2.5-4.6)$ & $2.6(2-4)$ \\
\hline Bilateral testicular volume $\left(\mathrm{cm}^{3}\right)$ & $15.5(14-19)$ & $16.0(15-20)$ & - \\
\hline
\end{tabular}

Abbreviations: E2, estradiol; FSH, follicle-stimulating hormone; LH, luteinizing hormone; PRL, prolactin; T, testosterone.

${ }^{a}$ Reference values $=2-10$;

${ }^{\mathrm{b}}$ Reference values $=0.8-10.0$;

${ }^{\mathrm{c}}$ Reference values $=221.8-786.2$;

${ }^{\mathrm{d}}$ Reference values $=8-40$;

e Reference values $=20-75$;

${ }^{\mathrm{f}}$ Data are presented as median (range);

$\infty=$ infinite; - , not tested. 
Saylam et al. ${ }^{4}$ did not mention FSH levels in their study. One of our patients had a high $(>10) \mathrm{T} / \mathrm{E} 2$ ratio, and the ratio was low in the other three patients (Table 1). Thus, at present, it is unknown whether a normal blood FSH level or a low T/E2 ratio is more predictive of spermatogenesis improvement in NOA.

It is unlikely that letrozole treatment increases congenital malformations. In fact, no malformed babies were fathered by infertile males in the course of this treatment. ${ }^{4,12,13}$ Additional studies have also shown that letrozole does not increase the risk for congenital malformations with ovulation induction. ${ }^{14}$

If our data are confirmed, this and future research may save some NOA patients from surgery. Future research in this field should also consider the possibility that letrozole administration could improve sperm yield in TESE/FNA and/or sperm retrieval for intracytoplasmic sperm injection. The latter is strongly influenced by even a minimal improvement in spermatogenesis. ${ }^{15}$

\section{AUTHOR CONTRIBUTIONS}

GC wrote the paper and studied two cases. Giovanni Beretta and Giulio Biagiotti studied one case each.

\section{COMPETING FINANCIAL INTERESTS}

The authors declare no competing financial interest.

1 Donoso $\mathrm{P}$, Tournaye H, Devroey $\mathrm{P}$. Which is the best sperm retrieval technique for nonobstructive azoospermia? A systematic review. Hum Reprod Update 2007; 13: 539-49.
2 Biagiotti G, Cavallini G, Modenini F, Vitali G, Gianaroli L. Spermatogenesis and spectral echo-colour Doppler traces from the main testicular artery. BJU Int 2002; 90: 903-8.

3 Patry G, Jarvi K, Grober ED, Lo KC. Use of the aromatase inhibitor Letrozole to treat male infertility. Fertil Steril 2009; 92: 829.

4 Saylam B, Efesoy O, Cayan S. The effect of aromatase inhibitor Letrozole on body mass index, serum hormones, and sperm parameters in infertile men. Fertil Steril 2011; 95: 809-11.

5 McArthur HL, Morris PG. Aromatase inhibitor strategies in metastatic breast cancer. Int J Womens Health 2010; 1: 67-72.

6 Swanton A, Itani A, McVeigh E, Child T. Azoospermia: is sample centrifugation indicated? A national survey of practice and the Oxford experience. Fertil Steril 2007; 88: 374-8.

7 Lin CC, Huang WJ, Chen KK. Measurement of testicular volume in smaller testes: how accurate is the conventional orchidometer? J Androl 2009; 30: 685-9.

8 de Krester DM, Kerr JB. The cytology of the testis. In: Knobil E, Nell JD, editors. The Physiology of Reproduction. New York: Raven Press; 1988. pp837-932.

9 Camussi A, Moller F, Ottaviano E, Gorla M. Metodi statitistici per la sperimentazione biologica. 2nd ed. Bologna: Zanichelli; 1995.

10 Carani C, Rochira V, Faustini-Fustini M, Balestrieri A, Granata AR. Role of oestrogen in male sexual behaviour: insights from the natural model of aromatase deficiency. Clin Endocrinol 1999; 51: 517-24.

11 Rugo HS. The breast cancer continuum in hormone-receptor-positive breast cancer in postmenopausal women: evolving management options focusing on aromatase inhibitors. Ann Oncol 2008; 19: 16-27.

12 Raman JD, Schlegel PN. Aromatase inhibitors for male infertility. J Urol 2002; 167 624-9.

13 Roth MY, Amory JK, Page ST. Treatment of male infertility secondary to morbid obesity. Nat Clin Pract Endocrinol Metab 2008; 4: 415-9.

14 Badawy A, Elnashar A, Totongy M. Clomiphene citrate or aromatase inhibitors combined with gonadotropins for superovulation in women undergoing intrauterine insemination: a prospective randomised trial. J Obstet Gynaecol 2010 30: 617-21.

15 Cavallini G, Magli MC, Crippa A, Resta S, Vitali G et al. The number of spermatozoa collected at testicular sperm extraction is a novel predictor of intracytoplasmic sperm injection outcome in non-obstructive azoospermic patients. Asian J Androl 2011; 13: 312-6. 